\section{Sobre COVID-19, estrés y el cerebro de los adolescentes}

\section{On COVID-19, stress and teenagers' brain}

\section{Señor Editor,}

La interesante revisión de Cuadra-Martínez y colaboradores $^{1}$ destaca la necesidad de comprender el comportamiento psicológico durante las pandemias, dimensión que debe orientar las medidas políticas de control y las intervenciones psicológicas y de salud en general, de modo que sean efectivas para el manejo de la pandemia por COVID-19. Un grupo etario psicológicamente vulnerable este contexto son los adolescentes ${ }^{2}$. La adolescencia conlleva profundos cambios en los sistemas fisiológicos y neuroendocrinos, junto con la reorganización de los sistemas neuronales necesarios para una adecuada función ejecutiva, procesamiento socioemocional y regulación de las emociones ${ }^{3}$. Los adolescentes, por tanto, se encuentran en un estadio crítico de desarrollo neurobiológico, de alta neuroplasticidad, que puede ser afectado por distintos factores es- tresores ${ }^{2,3}$. La presente carta tiene por objetivo comentar cómo el estrés ocasionado por COVID-19 puede afectar el desarrollo del cerebro adolescente.

Hace ya casi cuatro décadas ${ }^{4}$, el Dr. Luis Vargas Fernández (1912-2011), Premio Nacional de Ciencias, Miembro Honorario de la Sociedad Médica de Santiago y destacado endocrinólogo, rememoraba al ilustre escritor médico romano Celso (Aulus Cornelius Celsus; ca. 25 a.C.-50 d.C.), quien habría mencionado que «el miedo, la rabia y cualquier otro estado de la mente pueden a menudo ser capaces de alterar el pulso» ${ }^{4}$. Con esto, el Dr. Vargas daba un sustento histórico a un estado que concitó el interés de investigadores durante siglos, pero que recién fue conceptualizado durante el s. XX por los célebres fisiólogos Walter B. Cannon (1871-1945) y Hans Selye (1907-1982): el Estrés. Se define Estrés como la respuesta a estímulos que alteran la homeostasis bioquímica, inmunológica, fisiológica y psicológica del individuo ${ }^{4}$. Es un comportamiento heredado, defensivo y/o adaptativo que implica la activación específica de mecanismos neuroendocrinos ante estímulos (estresores) amenazantes ${ }^{4}$.

Varios meses de pandemia han expuesto a niños, jóvenes y adultos de todo el mundo a niveles de estrés 


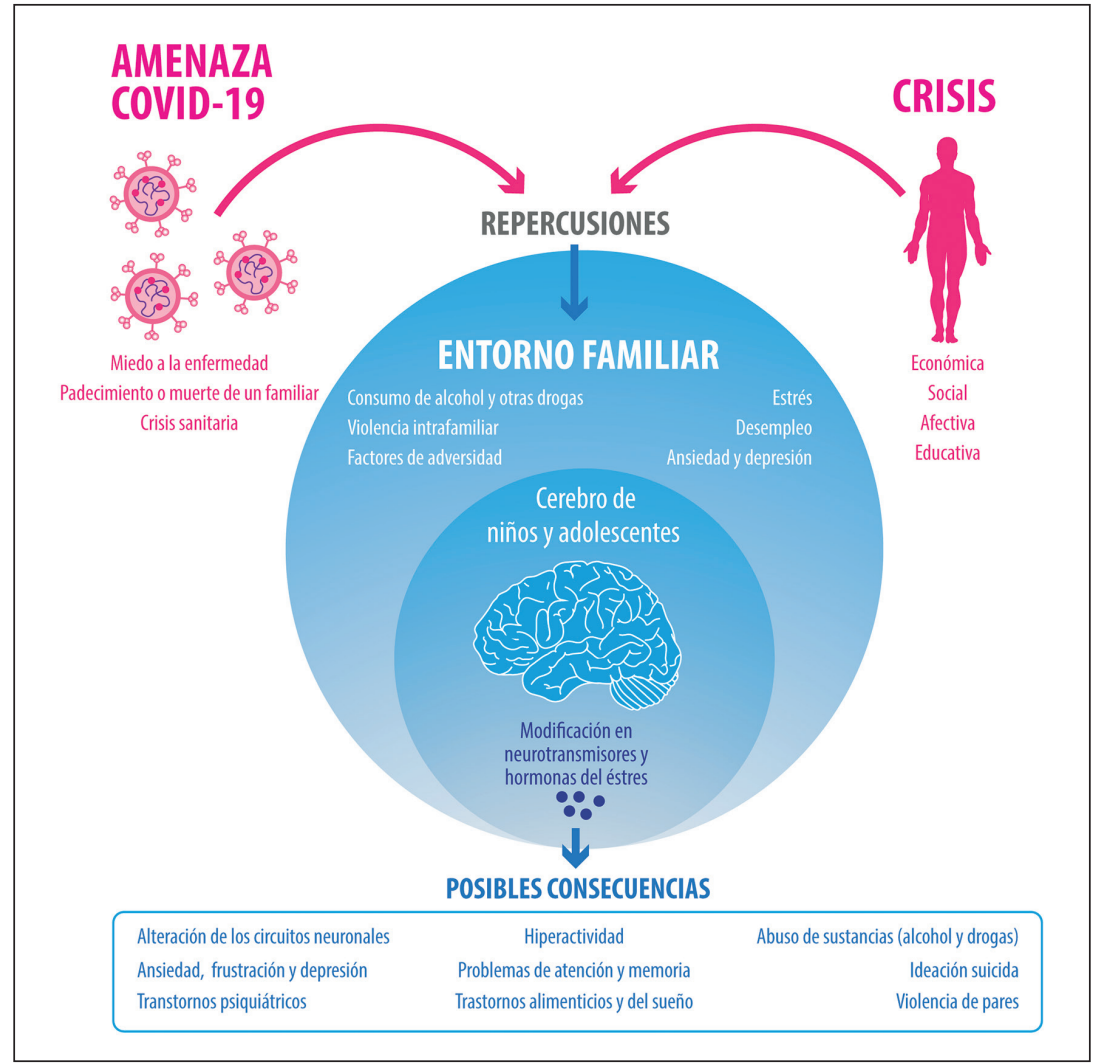

Figura 1. Posibles consecuencias entre los adolescentes debido a la amenaza de la pandemia por COVID-19 y las distintas crisis relacionadas con ella. Adaptado de Palacio-Ortiz et al. ${ }^{2}$.

variables, pero permanentes. Lo anterior, debido a muchos factores, entre ellos, el temor a contagiarse o que lo haga algún familiar y las cuarentenas y otras formas de restricción de la movilidad, impuestas para controlar el avance de COVID-19². Los sistemas educativos han sido fuertemente desafiados durante la pandemia. Los establecimientos educacionales de todos los niveles han permanecido cerrados o funcionando parcialmente, las clases han debido efectuarse de manera sincrónica o asincrónica mediante nuevas tecnologías de la información y comunicación (TICs) que implican el uso de pantallas y plataformas informáticas, conllevando esfuerzos de organización de horarios de clases y adaptación a dichas TICs por parte de los jóvenes, sus familias y los profesores, quienes también reportan estar estresados 5 . Por otra parte, los adolescentes han disminuido significativamente sus actividades de interacción social, el contacto cara a cara con sus pares y otras instancias de esparcimiento, diversión y actividad física, todas éstas fundamentales para la consolidación de la autoestima, la identidad personal y las habilidades sociales que sustentan el bienestar psicológico.

¿Cómo impacta el estrés en el cerebro de los adolescentes? En ellos el impacto del estrés es profundo, duradero y con dimorfismo sexual pues las hormonas esteroidales sexuales y las hormonas involucradas en la respuesta al estrés ( $c f r$. eje hipotalámico-hipofisario-adrenal) interactúan para dar forma a las futuras respuestas endocrinas. La activación de la respuesta al estrés finaliza con la secreción de las hormonas del estrés, e.g., glucorticoides como el cortisol (hidrocortisona) y la corticosterona (11, 21 -dihidroxiprogesterona), cuya acción induciría alteraciones genéticas y neuroquímicas en múltiples regiones del cerebro de los mamíferos ${ }^{6}$. En este sentido, investigaciones en animales sugieren que la secreción excesiva de corticosterona produce neurotoxicidad en áreas cerebrales ricas en receptores de glucocorticoides, e.g., hipocampo y corteza prefrontal ${ }^{6}$, las cuales participan en el procesamiento de la memoria y la función ejecutiva, ambas críticas para el proceso de aprendizaje.

Por otra parte, durante el desarrollo de la pandemia -y también una vez ésta finalice- los adolescentes pueden verse afectados por trastorno de estrés postraumático ${ }^{2}$, el cual implica alteraciones psicológicas duraderas atribuidas a la experiencia de un evento traumático importante, por ejemplo, la muerte de un familiar, su hospitalización o meses de confinamiento en sus hogares, sin poder socializar (Figura 1). Se sabe que el trastorno de estrés postraumático es frecuente y 
debilitante entre los adolescentes ${ }^{3}$ y no debe ser subestimado como una de las consecuencias de la pandemia.

Lo descrito permite concluir que el estrés generado en el contexto de COVID-19 puede afectar el desarrollo del cerebro adolescente, alterando distintos mecanismos neuropsicológicos. Debido a que la pandemia todavía está en curso, no se puede aún estimar el real impacto de estos efectos, pero lo cierto es que el abordaje de esta problemática requerirá indudablemente un enfoque multidisciplinario integral enfocado en la salud mental adolescente.

Manuel E. Cortés ${ }^{1,2, a}$

${ }^{1}$ Escuela de Educación Inicial y Escuela de Educación Diferencial, Facultad de Educación, Universidad Bernardo O'Higgins (UBO). ${ }^{2}$ Programas de Magíster en Ciencias Químico Biológicas $y$ Doctorado en Educación, UBO.

${ }^{a}$ Biólogo, Profesor de Química y Biología, Ingeniero en Prevención de Riesgos, $M B A, M S c, P h D$.

\section{Referencias}

1. Cuadra-Martínez D, Castro-Carrasco PJ, Sandoval-Díaz J, Pérez-Zapata D, Mora Dabancens D. COVID-19 y comportamiento psicológico: Revisión sistemática de los efectos psicológicos de las pandemias del siglo XXI. Rev Med Chile 2020; 148 (8): 1139-54.

2. Palacio-Ortiz JD, Londoño-Herrera JP, Nanclares-Márquez A, Robledo-Rengifo P, Quintero-Cadavid CP. Psychiatric disorders in children and adolescents during the COVID-19 pandemic. Rev Colomb Psiquiatr. 2020; 49 (4): 279-88.

3. Cisler JM, Herringa RJ. Posttraumatic stress disorder and the developing adolescent brain. Biol Psychiatry. 2021; 89 (2): 144-51.

4. Vargas L. Entorno íntimo y psiconeuroendocrinología del estrés crónico. Rev Chil Neuropsiquiatr. 1984; 22 (4): 25964.

5. Romero Jeldres M, Tenorio Eitel S. La Educación en Tiempos de Confinamiento: Perspectivas de lo Pedagógico. Santiago, Chile: Fondo Editorial UMCE, 2021.

6. Arbel I, Kadar T, Silbermann M, Levy A. The effects of long-term corticosterone administration on hippocampal morphology and cognitive performance of middle-aged rats. Brain Res. 1994;657(1-2):227-35.

Correspondencia

Dr. Manuel E. Cortés

Decano, Profesor Titular, Facultad de Educación, UBO,

C.P. 8370993, Santiago, Chile.

cortesmanuel@docente.ubo.cl 